\title{
Multicomponent stellar winds and chemical peculiarity in A stars
}

\author{
Jiří Krtička ${ }^{1}$ and Jiří Kubát ${ }^{2}$ \\ ${ }^{1}$ Ústav teoretické fyziky a astrofyziky PřF, Masarykova univerzita, CZ-611 37 Brno, Czech \\ Republic, email: krticka@physics.muni.cz \\ ${ }^{2}$ Astronomický ústav, Akademie věd České republiky, CZ-251 65 Ondřejov, Czech Republic, \\ email: kubat@sunstel.asu.cas.cz
}

\begin{abstract}
We calculate multicomponent radiatively driven stellar wind models suitable for A stars. We discuss the possible decoupling of individual elements from the stellar wind and its influence on the chemical peculiarity of these stars. We obtain a range of stellar parameters for different types of multicomponent flow.
\end{abstract}

Keywords. Hydrodynamics, stars: mass loss, stars: early-type, stars: winds

\section{Introduction}

Stellar winds of hot stars are accelerated mainly due to the absorption of radiation in the resonance lines of heavier elements like carbon, nitrogen, oxygen or iron, and due to light scattering by free electrons. Since the stellar wind is composed mostly of hydrogen and helium, the process of wind acceleration consists basically of two steps. In the first step momentum is transferred from the radiation field to heavier elements, which have enough absorbing lines. Passive components (hydrogen and helium) are accelerated in the following step due to Coulomb collisions with heavier elements (Castor et al. 1976). Clearly, stellar winds of hot stars have a multicomponent nature. For a relatively high density stellar wind, the multicomponent wind nature does not influence the wind structure, thus high-density stellar winds (e.g.,those of galactic $\mathrm{O}$ stars) may be adequately modelled as one-component ones. However, this is not the case for low-density stellar winds (Krtička \& Kubát 2001, hereafter KKII) for which the velocity difference between the wind components becomes comparable with the thermal speed. Many new physical effects occur in this case, which may influence the wind structure, ranging from frictional heating, wind decoupling, and hydrogen fallback to a pure metallic wind. Here we study multicomponent effects in the A star winds and discuss their consequences on the chemical peculiarity of these stars.

\section{Wind models}

\subsection{Model equations}

Published multicomponent wind models have been described in detail by KKII and Krtička (2003). Here we only summarize their basic properties. We assume a stationary and spherically symmetric stellar wind, which is composed of four components, namely metallic ions, hydrogen, helium, and free electrons. For the calculation of wind models we solve the continuity equation, the momentum equation, and the energy equation for each component of the flow. The continuity equation has the form

$$
\frac{\mathrm{d}}{\mathrm{d} r}\left(r^{2} \rho_{a} v_{r a}\right)=0
$$


where $\rho_{a}$ is the density, $r$ is the radius, and $v_{r_{a}}$ is the velocity of a component $a$. The momentum equation is

$$
v_{r a} \frac{\mathrm{d} v_{r a}}{\mathrm{~d} r}=g_{a}^{\mathrm{rad}}-g-\frac{1}{\rho_{a}} \frac{\mathrm{d}}{\mathrm{d} r}\left(a_{a}^{2} \rho_{a}\right)+\frac{q_{a}}{m_{a}} E+\sum_{b \neq a} g_{a b}^{\text {fric }},
$$

where $g_{a}^{\mathrm{rad}}$ is the radiative acceleration, $g$ is the gravity acceleration, $E$ is the electric polarisation field, and $g_{a b}^{\text {fric }}$ is the frictional force (Burgers 1969)

$$
g_{a b}^{\text {fric }}=\frac{1}{\rho_{a}} K_{a b} G\left(x_{a b}\right) \frac{v_{r b}-v_{r a}}{\left|v_{r b}-v_{r a}\right|},
$$

where $G\left(x_{a b}\right)$ is the Chandrasekhar function. The frictional coefficient is

$$
K_{a b}=n_{a} n_{b} \frac{4 \pi q_{a}^{2} q_{b}^{2}}{k T_{a b}} \ln \Lambda,
$$

where the mean temperature $T_{a b}=\left(m_{b} T_{a}+m_{a} T_{b}\right) /\left(m_{b}+m_{a}\right)$ is calculated using temperatures $T_{a}$ and $T_{b}$ of individual wind components with atomic masses $m_{a}$ and $m_{b}$. The radiative force in the CAK approximation (Castor et al. 1975) due to line-absorption acts on the metals and the radiative force due to Thomson scattering acts on free electrons.

The energy equation for each component of the flow is

$$
\frac{3}{2} v_{r a} \rho_{a} \frac{\mathrm{d} a_{a}^{2}}{\mathrm{~d} r}+\frac{a_{a}^{2} \rho_{a}}{r^{2}} \frac{\mathrm{d}}{\mathrm{d} r}\left(r^{2} v_{r a}\right)=Q_{a}^{\mathrm{rad}}+\sum_{b \neq a}\left(Q_{a b}^{\mathrm{ex}}+Q_{a b}^{\mathrm{fric}}\right),
$$

where $Q_{a b}^{\mathrm{ex}}$ is the heat exchange, $Q_{a b}^{\text {fric }}$ is the frictional heating and $Q_{a}^{\mathrm{rad}}$ is the radiative heating calculated using the thermal balance of electrons method (Kubát et al. 1999).

The system of hydrodynamic equations is closed using the equation for the electric polarisation field (see KKII) and by the equations of ionization equilibrium (we assume a nebular approximation, see Mihalas (1978), Eq. 5.46).

\subsection{The possibility of wind decoupling}

The frictional force Eq. (2.3) between two components depends on the velocity difference between these two components via the so-called Chandrasekhar function,

$$
G\left(x_{a b}\right)=\frac{1}{2 x_{a b}^{2}}\left[\Phi\left(x_{a b}\right)-x_{a b} \frac{\mathrm{d} \Phi\left(x_{a b}\right)}{\mathrm{d} x_{a b}}\right],
$$

where the dimensionless velocity difference $x_{a b}$ is given by

$$
x_{a b}=\frac{\left|v_{r b}-v_{r a}\right|}{\alpha_{a b}}=\frac{\Delta v_{a b}}{\alpha_{a b}},
$$

where $\alpha_{a b}$ is the mean thermal speed, $\alpha_{a b}^{2}=2 k\left(m_{a} T_{b}+m_{b} T_{a}\right) /\left(m_{a} m_{b}\right)$. A plot of the Chandrasekhar function is given in Figure 1. For relatively low velocity differences, $\Delta v_{a b} \lesssim \alpha_{a b}$, the Chandrasekhar function is an increasing function of the velocity difference $\Delta v_{a b}$ and components $a$ and $b$ are well coupled. However, for higher velocity differences, $\Delta v_{a b} \gtrsim \alpha_{a b}$, the Chandrasekhar function is a decreasing function of the velocity difference and the decoupling of the components may occur.

Important simplifications. Our models used some important simplifications which may affect the validity of results obtained, especially in the case of A stars:

- the radiative force is calculated in the CAK approximation with force multipliers after Abbott (1982), thus wind parameters may not be adequate, 


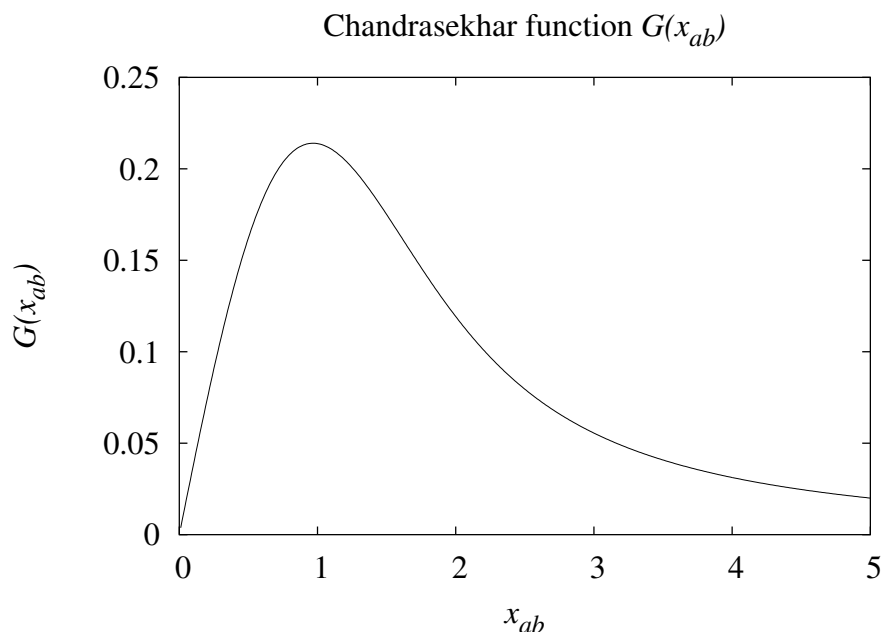

Figure 1. The run of the Chandrasekhar function. Note that Eq. (2.7) yields $x_{a b} \sim \Delta v_{a b}$, where $\Delta v_{a b}$ is the velocity difference between the wind components. If the flow is well coupled, $x_{a b} \lesssim 0.97, G\left(\Delta v_{a b}\right) \sim \Delta v_{a b}$. If the drift velocity is large, $x_{a b} \gtrsim 0.97, G\left(\Delta v_{a b}\right) \sim \Delta v_{a b}^{-2}$, and the wind may decouple. Note that the point $x_{a b} \approx 0.97$, corresponds to the maximum of $G$.

- the ionization equilibrium is approximated using a "nebular approximation" after Mihalas (1978), this may influence the frictional force,

- we neglect wind instabilities (Owocki \& Puls 1999),

- we neglect magnetic fields (ud-Doula \& Owocki 2002),

- only Coulomb collisions are accounted for the calculation of the frictional force.

\section{Multicomponent wind models}

\subsection{Types of hot star winds}

There are several types of hot-star winds with respect to multicomponent effects:

- winds with negligible multicomponent effects (these winds may be adequately described by one-component models, e.g., winds of galactic O supergiants),

- winds with the temperature influenced by frictional heating (e.g., winds of MainSequence B stars),

- winds where the decoupling of the components occurs (which may result in helium decoupling or in both hydrogen and helium decoupling),

- the decoupling of wind components in the atmosphere (i.e., at the onset of the wind); for helium decoupling, a helium-free wind may exist, this may cause a helium overabundance in the atmosphere; for the case of both hydrogen and helium decoupling, a pure-metallic stellar wind may be present.

\subsection{Helium decoupling}

Helium decoupling was proposed by Hunger \& Groote (1999) as the explanation of chemical peculiarity of Bp stars. Helium decoupling in the atmosphere occurs when the helium frictional acceleration is lower than the absolute value of gravity acceleration acting on helium,

$$
g_{\alpha \mathrm{p}}^{\text {fric }}<g
$$




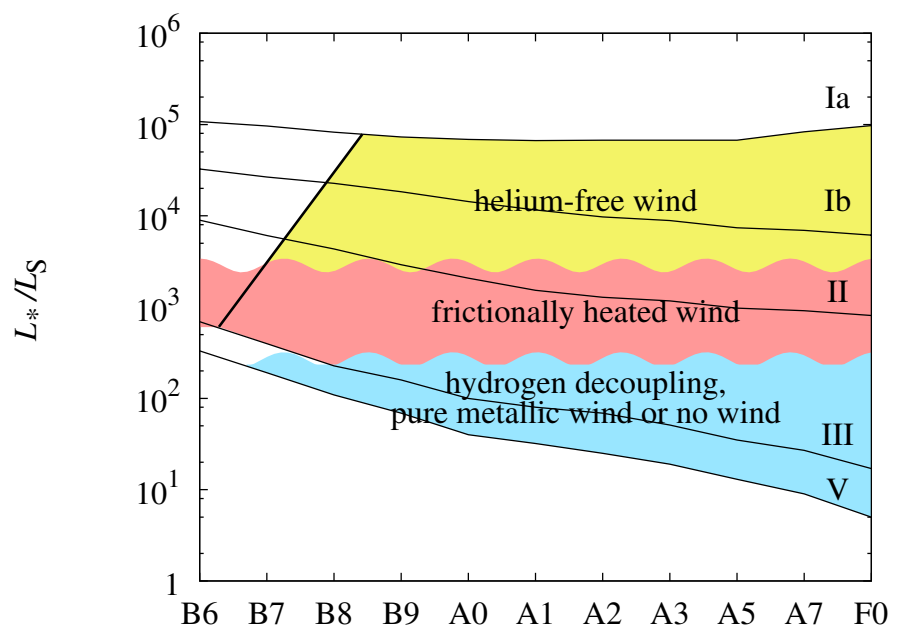

Figure 2. The domains in HR diagram where stars with different types of stellar wind exist. The fundamental stellar parameters of individual spectral types are taken from Straižys \& Kuriliene (1981).

thus, for solar metallicity stars helium decouples when the mass-loss rate is lower than

$$
\dot{M} \lesssim 2 \cdot 10^{-16} M_{\odot} \operatorname{year}^{-1}\left(\frac{M}{M_{\odot}}\right)\left(\frac{T_{\text {eff }}}{10^{4} \mathrm{~K}}\right)^{3 / 2} z_{\alpha}^{-2} .
$$

which can easily be found from the Eq. (3.1) using Eqs. (2.3) and (2.4).

In A stars helium may be neutral in the stellar atmosphere, thus it is not sufficiently accelerated by friction, hence a helium-free wind may be present in this case (see Figure 2). Consequently, some A stars may have an enhanced abundance of helium in their atmospheres. However, this effects occurs for late B stars outside the domain of the $\mathrm{He}$ rich B stars, consequently our models do not support the model of helium enrichment in the atmospheres of He rich B stars proposed by Hunger \& Groote (1999).

\subsection{Frictional heating}

Multicomponent effects are found to be important if the velocity difference is comparable with the thermal speed (see Krtička et al. 2003),

$$
\frac{v_{r \mathrm{i}}-v_{r \mathrm{p}}}{\sqrt{\frac{2 k T}{m_{\mathrm{p}}}}} \gtrsim 0.1
$$

For solar metallicity stars multicomponent effects become important for mass-loss rates lower than

$$
\dot{M} \lesssim 10^{-10} M_{\odot} \operatorname{year}^{-1}\left(\frac{v_{\infty}}{10^{8} \mathrm{~cm} \mathrm{~s}^{-1}}\right)^{3}\left(\frac{R_{*}}{R_{\odot}}\right)\left(\frac{T_{\text {eff }}}{10^{4} \mathrm{~K}}\right) \frac{1}{z_{\mathrm{H}}^{2} z_{\mathrm{i}}^{2}} .
$$

In such a case the stellar wind may be heated by friction. An example of such frictionally heated wind of an A5 II star is given in Figure 3. The domain in HR diagram where stars with frictionally heated wind may exist is displayed in Figure 2.

Note that in a low-density stellar wind another type of heating, the so-called GayleyOwocki heating (Gayley \& Owocki 1994, KKII), may be important for the wind temerature balance. 

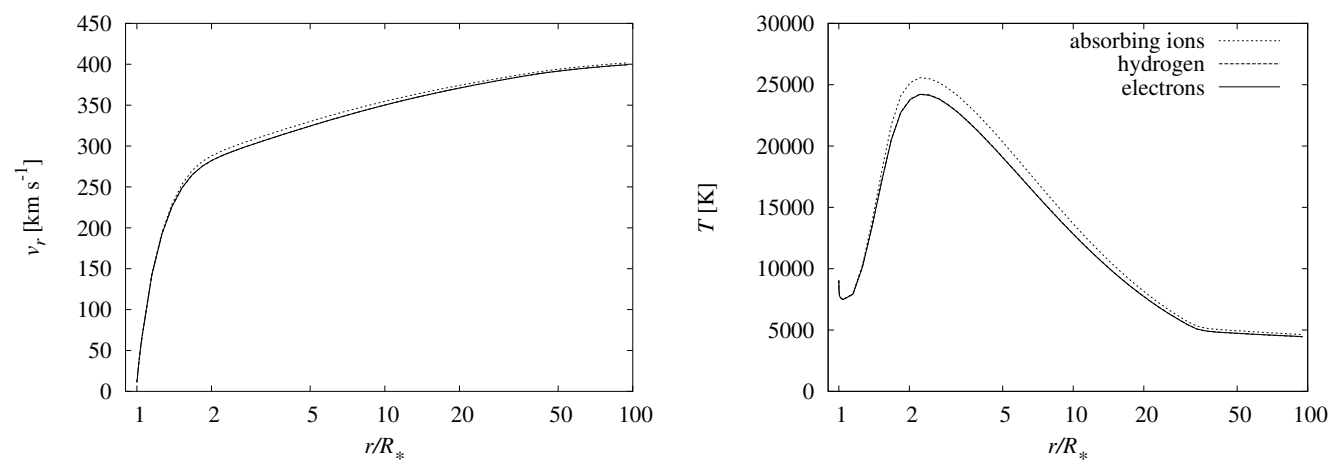

Figure 3. An example of the frictionally heated wind of an A5 II star $\left(T_{\text {eff }}=8300 \mathrm{~K}\right.$, $\left.M_{*}=5.5 M_{\odot}, R_{*}=15.1 R_{\odot}\right)$. Left panel: The velocities of the individual components of the stellar wind. Note that velocities of wind components are nearly equal. Right panel: The temperatures of the individual components. The stellar wind is frictionally heated around $r \approx 2 R_{*}$.
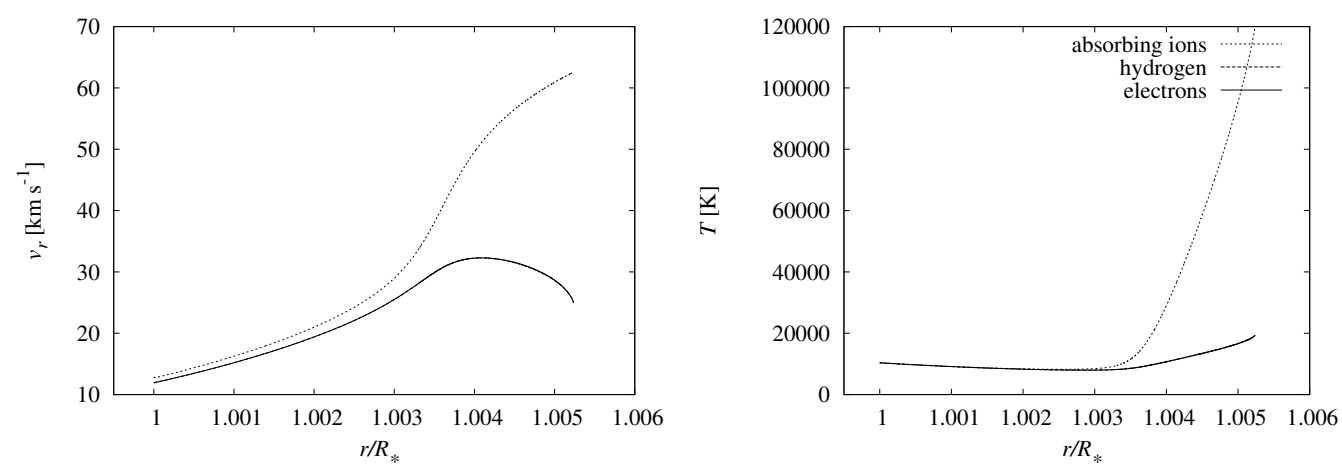

Figure 4. An example of hydrogen decoupling in the wind of an A0 III star $\left(T_{\text {eff }}=9600 \mathrm{~K}\right.$, $\left.M_{*}=2.7 M_{\odot}, R_{*}=3.63 R_{\odot}\right)$. Left panel: The velocities of the individual components of the stellar wind. Stellar wind decouples around $r \approx 1.004 R_{*}$ in this case the hydrogen component deccelerates. Right panel: The temperatures of the individual components. Note the strong frictional heating in the outer parts of the wind.

\subsection{Hydrogen decoupling}

Hydrogen decoupling occurs if the velocity difference is equal to the thermal speed,

$$
\frac{v_{r \mathrm{i}}-v_{r \mathrm{p}}}{\sqrt{\frac{2 k T}{m_{\mathrm{p}}}}} \approx 1 .
$$

After decoupling there are several possibilities for further hydrogen movement. Namely

- hydrogen leaves the star if the hydrogen velocity is greater than the escape velocity,

- hydrogen falls back onto the stellar surface or forms clouds above the surface (Porter \& Skouza 1999) if the hydrogen velocity is lower than the escape velocity,

- hydrogen decouples in the atmosphere, only a pure metallic wind exist (Babel 1995, 1966) if the frictional force is small already in the stellar atmosphere.

An example of hydrogen decoupling in the wind of an A0 III star is given in Figure 4. The domains in HR diagram where stars with hydrogen decoupling, a pure metallic wind or without any wind may exist are displayed in Figure 2. 


\section{Conclusions and discussion}

We have shown that multicomponent effects may be very important for those A stars which have a stellar wind. We have indicated the domains of different types of multicomponent stellar wind in the HR diagram (see Figure 2). First, it is possible that for A stars helium decouples from the stellar wind already in the stellar atmosphere and, consequently, that A stars have helium-free winds. Secondly, for stars with lower luminosities than those of supergiants, frictional heating may influence the wind temperature. Stars with even lower luminosities have a fractionated stellar wind due to hydrogen decoupling. Finally, A stars with the smallest luminosities have a pure metallic wind or do not have a stellar wind at all.

However, the detailed location of stars with different types of stellar wind in HR diagram depends on the model assumptions, especially on ionization equilibrium and wind parameters. Consequently, more advanced (NLTE, see Krtička \& Kubát, 2005) models are necessary to study these effects in detail.

\section{Acknowledgements}

This work was supported by grants GA ČR 205/02/0445, 205/03/D020, 205/04/1267, MVTS SR-CR 128/04. JKr is grateful to IAU for travel grant. The Astronomical Institute Ondřejov is supported by projects K2043105 and Z1003909.

\section{References}

Abbott, D. C. 1982 ApJ 259, 282-301

Babel, J. 1995 AESA 301, 823-839

Babel, J. 1996 A $\& A$ 309, 867-878

Burgers, J. M. 1969 Flow equations for composite gases, Academic Press, New York

Castor, J. I., Abbott, D. C., \& Klein, R. I. 1975 ApJ 195, 157-174

Castor, J. I., Abbott D. C., \& Klein R. I., 1976 In Physique des mouvements dans les atmosphères stellaires (eds. R.Cayrel \& M.Sternberg) CNRS Paris, p.363

Gayley, K. G. \& Owocki, S. P. 1994 ApJ 434, 684-694

Hunger, K., \& Groote, D. 1999 A\& A, 351, 554-558

Krtička, J. 2003 In Stellar Atmosphere Modelling (eds. I. Hubeny, D. Mihalas \& K. Werner) ASP Conf. Ser., Vol. 288, 259-262

Krtička, J. \& Kubát, J. 2001 A $\& A 377$ 175-191 (KKII)

Krtička, J. \& Kubát, J. 2005, These Proceedings, 23

Krtička, J., Owocki, S. P., Kubát, J., Galloway, R. K., \& Brown, J. C. 2003 A\&SA, 402, 713-718

Kubát, J., Puls, J. \& Pauldrach, A. W. A. 1999 A $\&$ A 341, 587-594

Mihalas, D. 1978 Stellar Atmospheres, 2nd ed., W. H. Freeman \& Comp., San Francisco

Owocki, S. P., \& Puls, J. 1999 ApJ 510, 355-368

Porter, J. M., \& Skouza, B. A. 1999 A\& A 344, 205-210

Straižys, V., \& Kuriliene, G. 1981 Ap\&SS 80, 353-368

ud-Doula, A., \& Owocki, S. P. 2002 ApJ 576, 413-428

\section{Discussion}

LANDSTREET: Your slide shows metallic or no winds for late B and early A Main Sequence stars. Do you have models yet to predict whether winds occur in such stars, and how the mass loss rate would vary with spectral type?

KRTIČKA: Unfortunatelly, we do not have such models yet. However, this is an interesting problem and we plan to calculate these models in the future.

VAUCLAIR: In case of a decoupled wind in A stars, with helium remaining behind, I think 
that helium would accumulate below the atmosphere, at the place of the helium ionization (Cunha et al. 2005, These Proceedings, 359) while it would occur in the atmospheres of B stars, around $T_{\text {eff }}=20000 \mathrm{~K}$, where helium rich stars are actually observed.

KRTIČKA: Yes, I agree. We would like to mention that our models do not support the explanation of peculiar helium abundances in some B stars proposed by Hunger \& Groote (1999) and probably some diffusion mechanism (as you discuss above) shall be invoked.

PRESTON: Does your formalism permit the calculation of the removal of metallic dust grains in the cooler (RV Tauri) stars?

KRTIČKA: Yes, it would be possible to include a dust grain component into our models. As we have calculated wind models of hot stars only, we have not included it. 